\title{
EL HILO DE ARIADNA. EL MÉTODO DE ATRIBUCIÓN A PINTORES EN LA CERÁMICA ÁTICA
}

\author{
POR \\ CARMEN SÁNCHEZ \\ Departamento de Historia y Teoria del Arte UAM. Madrid.
}

\section{RESUMEN}

La forma de atribución a pintores anónimos de los vasos áticos fue inventada por Beazley transformando el método aplicado a la pintura italiana primero por Morelli y luego por Berenson. En este trabajo se intenta analizar cómo y cuándo ha surgido el método, en qué consiste, cómo se emplea, cuáles son sus perspectivas y dónde están sus limitaciones.

\section{SUMMARY}

It was Beazley who first applied a method of attributing anonimous painters to Attic Pottery. This method was an adaptation of that conceived by Morelli and Berenson for Italian Painting. I analized in this paper when and why it was put to practice, what are its presupositions, how it is applied, how it was criticized and the after Beazley prospectives.

«No veo más de lo que ven todos, pero me he adiestrado en fijarme en lo que veom. (Sherlock Holmes), Conan Doyle, The case book of Sherlock Holmes.

Todos aquellos que se han ocupado o nos ocupamos en el estudio de la cerámica griega nos enfrentamos cotidiana e inevitablemente al hecho de tener que atribuir un vaso a un grupo o a un pintor determinado. El trabajo resulta complejo y laborioso. El aprendizaje, dificil. Tengo la intención de abordar aquí el tema de la atribución de vasos no firmados a pintores concretos, cómo y cuándo ha surgido y se ha desarrollado el método, en qué consiste, cómo se emplea, cuáles son sus perspectivas, dónde están sus limitaciones. Mi esfuerzo no conseguirá hacer más fácil el camino a los que lo elijan, pero espero que lo encuentren menos incierto.

Los vasos griegos por su gran calidad artística y por el atractivo de sus complejas escenas narrativas atrajeron desde muy pronto la atención de los estudiosos. Desde el siglo xviII, con los descubrimientos de las tumbas etruscas, y sobre todo en el xix, con la formación de las grandes colecciones en los museos europeos, los investigadores se fueron interesando cada vez más por el estudio de la cerámica ática.

Lo primero que fascinó a los eruditos de los siglos xviII y principios del xix fueron las escenas mitológicas que decoraban muchos vasos griegos, imágenes que permitian "ver" las narraciones de textos griegos y latinos; pero muy pronto los vasos griegos pasaron a ser objetos coleccionables por su belleza intrinseca. Los expertos comenzaron a aproximarse a su estudio asumiendo de forma natural los métodos de investigación propios de los historiadores del Arte. Así, a lo largo del siglo xx, el estudio estilistico, la precisión cronológica, la atribución a pintores y el análisis evolucionista del desarrollo de la cerámica ática de figuras negras y rojas han sido, y en gran parte siguen siendo, las lineas principales de la investigación en este campo. Esta forma de ver y de acercarse al estudio de la cerámica se debe en gran medida a los trabajos que desarrolló John Davidson Beazley, que sentó las bases del método atribucionista y que compiló en sus obras inmensas listas de pintores de vasos. Su clasificación y atribuciones deben mucho al método positivista, taxonómico, de la historia del Arte de Morelli.

Gracias al trabajo precursor de Beazley a lo largo de este siglo se ha avanzado de forma espectacular en el conocimiento de la cerámica ática y de los pintores de vasos. Centenares de pintores han sido identificados y millares de vasos atribudos. A pesar de todo, son aún muchos los vasos que no se han relacionado con un artista y muchos los pintores que 
no se han reconocido. La laguna es más grande en el ultimo periodo de figuras rojas, justo antes del cese de la producción de vasos pintados en Atenas. Beazley abandonó en parte el estudio de estos vasos del último periodo, de baja calidad artistica y de temas y ejecuciones repetitivos, donde el método quizà produzca un resultado menos brillante. Sin embargo en la actualidad cada vez se valora y se reivindica más su estudio !.

El método de Beazley fue y aún es criticado. Muchos piensan que otras aproximaciones cientificas son quizá más adecuadas para el estudio de la cerámica antigua y descalifican abiertamente el método, como algunos historiadores del Arte hacen con el conocimiento pericial, al que consideran "como una actividad intrinsecamente conservadora y provista de anteojeras que se limita a reforzar el mercado del arte y eludir los problemas importantes centrándose en minucias particulares e insignificantes $)^{2}$, pero el mismo autor continúa un poco después afirmando que el conocimiento pericial -como el método de Beazley-merece ser analizado de cerca y no descalificado. No podemos negar que, gracias al trabajo de Beazley, se han conseguido importantes avances, en absoluto insignificantes en nuestro conocimiento de la Grecia antigua, y no sólo en el estudio de la cerámica ática. El método que inició Beazley ha sido tomado por otros autores y aplicado a otros tipos cerámicos antiguos. Así Trendall ha hecho una labor tan enorme como la de Beazley en el estudio de la cerámica de Italia y Sicilia ${ }^{3}$, Amyx y Benson en el de la cerámica corintia ${ }^{4}$, se ha aplicado también a la cerámica laconia ${ }^{5}$, etc.

Queremos aqui hacer una serie de reflexiones sobre el método, criticado pero aún en vigor, cuyo valor y resultados no deben despreciarse aunque otras aproximaciones al estudio de los vasos áticos sean simultáneamente posibles.

\footnotetext{
1J. Boardman, Athenian Red Figure Vases. The Classical Period, Londres, 1989 , p. 7 y 10.

21. Gaskell. Historia de las imágenes, en P. Burke (ed.) Formas de hacer Historia, Alianza, Madrid, 1993, (trad. de New Perspectives on Historical Writing, Polity Press, 1991), p. 216.

'Trendall, The Red-figured vases of Lucania. Campania and Sicily, Oxford, 1967: Id. The Red-figured Vases of Paestum, Londres, 1987; Trendall y Cambitoglou, The Red-figured Vases of Apulia, 2 vols., Oxford, 1978 y 1983. Una última sintesis en Red-figure Vases of South Iraly and Sicily, Londres, 1989.

${ }^{4}$ D. A. Amyx, Corinthian Vase-painting in the Archaic Period, 3 vols., 1989; J. L. Benson, Earlier corinthian workshops. A Survey of Corinthian Geometric and Protocorinthian Stylistic Groups, Amsterdam, 1989.

'P. E. Stibbe, C. M. Lakonische Vasenmaler des sechsten Jahr. hunderts v. Chr., Amsterdam, 1972.
}

\section{EL METODO DE BEAZLEY}

Las atribuciones de vasos. una práctica tan frecuente ahora, no siempre fue habitual. Jean de Witte en 1836. cuando el estudio de los vasos griegos estaba en sus albores y ni siquiera se diferenciaban producciones áticas de otras, como las etrusco-itálicas, fue el primero en señalar que dos vasos eran del mismo estilo. Más tarde, otros autores, como Klein, continuaron en la segunda mitad del siglo XIS las tentativas de agrupar los vasos firmados, siguieron Hauser y sobre todo A. Furtwängler que fue el primero en catalogar los vasos en un museo ordenados por fábrica, periodo y estilo *. Pero estos primeros intentos de estudiar las enormes colecciones que reunian los museos, producto de las excavaciones del xix, según su estilo $y$ darles una atribución, quedan restringidos, con pocas excepciones, a los vasos firmados.

Realmente el inicio y desarrollo del método de la atribución se debe a John D. Beazley (1885-1970), profesor de Arqueologia Clásica y Arte en Oxford desde 1925 hasta 1956. Fue el primero en atribuir vasos no firmados a pintores ya conocidos y en reunir vasos que se asemejaban entre si por su estilo y eran a su vez diferentes de otros. La mejor forma de explicar estas semejanzas en el dibujo era suponer que representaban el trabajo de un artista concreto? ${ }^{?}$. Estos conjuntos son bautizados por Beazley con un nombre convencional dado al pintor anónimo. Los nombres se toman del lugar de conservación del "vase name», del tema iconográfico representado, de la forma del vaso, del nombre del yacimiento, de alguna peculiaridad en las figuras representadas, etc. Un gran paso se ha dado al desvincular la mano de un artista de la de un individuo histórico concreto que firmaba sus vasos.

En su primer trabajo en 1908 el estudioso inglés aún continúa la tradición heredada y no aborda el estudio de artistas. Luego, cuando publica su articulo «Kleophrades», reúne varios vasos clasificados por su estilo como pertenecientes a una misma mano $^{8}$. En este caso se conoce el nombre del alfarero pero no del pintor, pero eso, el nombre, es lo menos importante y lo que nos interesa no es conocerlo sino reconocer el pintor y su estilo ${ }^{\circ}$. A partir de aquí

\footnotetext{
'D. von Bothmer, The Amasis Painter and his world, Malibu y Londres, 1985, p. 35., V. Robertson, The art of vase-painting in Classical Athens, Cambridge, 1992, p. 3.

'Beazley, "Citharoedus", JHS, 42, 1922, p. 90.

"Beazley "Kleophrades", JHS, 30, 1910, p. 38-68.

"Beazley, "The Master of the Berlin amphora", JHS, 31, 1911. p. 276-295.
} 
Beazley sigue publicando y atribuyendo vasos a artistas anónimos. Asi en $1911^{\prime \prime \prime}$, reúne 38 vasos del que empieza llamando el Maestro del ánfora de Berlín, por un vaso de este museo y acaba llamándose el «Berlin Painter» como el anterior pasará a ser el «Kleophrades Painter». El método atribucionista de Beazley, fuertemente criticado por algunos al principio ", se termina por imponer y con la publicación en 1942 del Attic-Red figure painters y en 1956 de su compañero Atric-Black figure Painters. Beazley se convierte durante medio siglo en la máxima autoridad mundial en el estudio de los vasos áticos.

Pero, ¿de dónde surge la idea de Beazley? El método debe mucho a la obra iniciada por Morelli y continuada por Berenson. Morelli fue el primero, si no en utilizar el método de atribución, si en hacerlo público. En 1880 publicó en alemán bajo pseudónimo su Pintores italianos. Estudio critico sobre su trabajo. El llamado padre de la moderna Historia del Arte fue también el primero en utilizar la fotografia sistemáticamente como instrumento de estudio. El método atribucionista nace ligado a la fotografia, que permite comparar en la mesa de trabajo las distintas obras simultáneamente. Morelli tuvo contactos con la Arqueologia Clásica a través de Jean Paul Richter. La correspondencia entre ambos fue publicada por las hijas de este último ${ }^{12}$. Es sorprendente quizá, como señala $\mathrm{Kurtz}^{13}$, que la célebre hija de Jean Paul Richter, Gisella, después conservadora en el Metropolitan de Nueva York, no introdujera el método aplicado a los vasos griegos, aunque fue una de las primeras en admitir públicamente la importancia de la contribución de Beazley.

Bernard Berenson era ya muy conocido en Londres cuando Beazley se graduó. El joven inglés conoció la obra de Berenson, dado que estaba interesado por la pintura italiana, de la que tenia grandes conocimientos, asi como por la pintura flamenca ${ }^{14}$. Probablemente Berenson, veinte años mayor que Beazley e interesado a su vez por el Arte Clásico, conoció también su trabajo. Berenson recorrió todos los museos y galerias de Europa y América tomando notas y apoyando su trabajo con fotografías, pero, a diferencia de Beazley y de

\footnotetext{
${ }^{10}$ Véase (nota 9).

"Kurtz, "Beazley and the connoisseurship of Greek Vases" en Greek Vases, II, 1985, p. 240.

${ }^{12} \mathrm{~V}$. 1. Richter y G. Richter, Italienische Malerei der Renaissance im Briefweschel von G. Morelli and J. P. Richter, BadenBaden, 1960.

${ }^{13}$ Kurtz, Beazley and the connoisseurship... p. 241.

${ }^{14}$ Cf. B. Ashmole, "Sir John Beazley (1885-1970)", en Beazley and Oxford, Donna Kurtz (ed.), Oxford, 1985, p. 59.
}

Morelli, nunca dibujón ${ }^{12}$. A su muerte sus notas y fotografias pasaron a la Universidad de Harvard como las de Beazley pasarán a Oxford.

No es extraño encontrarnos en los escritos de Bea/ley y de otros expertos referencias a la pintura italiana e incluso comparaciones puntuales con los pintores del Cerámico, referencias y comparaciones que nos indican cómo el método de Beazley es hijo y deudor, a veces demasiado, del aplicado a la pintura italiana. Por ejemplo, de las figuras del Pintor de Kleophrades Beazley escribe que «colocan firmemente los pies más separados que las de Euthymides, una actitud que nos recuerda a Signorellin ${ }^{16}$. Otros autores como Gisella Richter comparan explicitamente el método de atribución de vasos: "Las personalidades individuales se destacan tan claramente como en la pintura del Renacimiento y se distinguen del mismo modon ${ }^{17}$.

El estudioso inglés trabajó en gran medida condicionado por su conocimiento de los pintores italianos y del funcionamiento de sus escuelas y talleres. Sobre todo en sus primeros articulos se puede observar esta dependencia de Beazley del método aplicado a la pintura italiana. Como bien señala Robertson ${ }^{18}$ la influencia directa de Morelli y Berenson se muestra en la utilización de la palabra «Master» para sus pintores anónimos. En su trabajo sobre el "Maestro» de Berlin ${ }^{19}$ aísla un grupo de vasos y los califica de "school-pieces», como «directas y conscientes imitaciones». Robertson piensa que quizá Beazley tenia en la cabeza de una forma demasiado modélica el funcionamiento del estudio de un pintor italiano como el «de Boticelli o Bellini donde aprendices y asistentes producian obras de los diseños del maestro" ${ }^{20}$. Esto ha suscitado una de las principales críticas al método, como veremos después.

Aún en trabajos recientes los origenes y la dependencia del método se hacen explicitos. Por ejemplo Williams no se resiste a la tentación de comparar algunos pintores griegos con los del quattrocento en su estudio sobre el dibujo de la figura humana en las dos primeras generaciones de pintores de figuras rojas ${ }^{21}$.

\footnotetext{
${ }^{15}$ Kurtz, Beazley and the connoisseurship... p. 243, nota 40.

${ }^{10}$ Beazley, cit. (n. 8), p. 42-43

${ }^{17} \mathrm{G}$. Richter, Attic red-figured Vases, 1946, p. 2.

${ }^{1 *}$ The art of vase-painting in classical Athens, Cambridge, 1992, p. 5

${ }^{19}$ "The Master of the Berlin amphora", JHS, 1911, p. $276-$ 295.

${ }^{20}$ "Beazley and Attic Vase-Painting", en Beazley and Oxford. Donna Kurtz (ed.), Oxford, 1985, p. 26.

${ }^{21}$ "It is tempting to compare the way that the generation following the Italian pioneers Brunelleschi, Masaccio and Do-
} 
Los pintores italianos del Renacimiento y los pintores de vasos de Atenas tienen muchos puntos en común. su preocupación por el dominio del espacio o su interés por la figura humana; dos momentos históricos lejanos en el tiempo y en el espacio reunidos al ser analizados bajo la luz de un mismo método.

Pero, ¿en qué consiste el método?

\section{ENTRE EL DETECTIVE Y EL PSICOANALISTA}

Las bases son la observación y la comparación. Kurtz nos recuerda que las bases del método de Morelli son «el cuidadoso escrutinio de los detalles, especialmente de aquellos que un artista reproduce tan regularmente que pueden ser considerados tan característicos de su mano como su escritura»" 22 La forma de razonar que utilizan Beazley, o MoreIli, es la misma lógica de la inducción que empleó ya el Zadig de Voltaire -publicado en 1747 método llegó a convertirse en la piedra angular de lo que a veces se denomina ciencia conjetural». El modo de razonar es común a varias ciencias, como la Geologia, la Arqueología, la Medicina, la Literatura o la Historia del Arte ${ }^{23}$. A la científica preocupación positivista se añade un pensamiento más humanista, un interés por el hombre como individuo. una curiosidad por rastrear, a través de una serie de indicios, la huella de una persona concreta.

El método que aplicó Morelli, o Beazley, es similar al de un buen médico, cuyo reconocimiento y valoración de pequeñas distinciones es el factor esencial para un diagnóstico acertado.

Mediante la observación y reconocimiento de los pequeños detalles variables, Beazley puede atribuir un vaso no firmado a un pintor anónimo. Es necesario encontrar las peculiaridades del pintor, descubrir sus hábitos inconscientes, involuntarios,

natello applied and experimented further with the discoveries of their predecessors to the situation I have been sketching in the Keramaikos at Athens. I do not mean to set Onesimos and Douris, the Brygos Painter and the Antiphon Painter, alongside Ucello and Pollaiuolo, Mantegna and Piero della Francesca, but there is perhaps something of the same bravado of pose and mouvement, brought about by a complete confidence of technique and a deep interest in the human body" Dyfri Williams, "The Drawing of the Human Figure on Farly Red-figure Vases", en New Perspectives in Early Greek Art. Buitron- Oliver (eds.), Hanover y Londres, 1991, p. 293.

${ }^{22}$ V. Kurtz, "Beazley and the connoisseurship..." p. 237.

${ }^{23} \mathrm{Cf}$. M. Sheperd, Sherlock Holmes y el caso del Dr. Freud. Zaragoza, 1990 , p. 19. incluso inadecuados y prestarles cuidadosa atención para un diagnóstico exacto. Este es el método que aplicó a la pintura italiana el doctor Giovanni Morelli. Otro médico aplicó la misma forma de razonar, Conan Doyle, pero puesto en boca de un inmortal personaje de fícción. Sherlock Holmes. Los paralelismos entre las enseñanzas de Morelli y los métodos de Sherlock Holmes ya han sido señalados por muchos autores ${ }^{24}$. Se trata en ambos casos de descubrir, a partir de pequeños indicios, desapercibidos para los no iniciados, el autor de una pintura o de un crimen. El método del detective - observación e inferencia - es similar al del médico que a través del reconocimiento de los sintomas, diagnostica.

Aplicado a desvelar los complejos mecanismos de la mente humana, Sigmund Freud desarrolló la técnica del psicoanálisis. El mismo señaló la relación íntima de esta técnica con los métodos aplicados por el Dr. Morelli a la Historia del Arte ${ }^{25}$. Lo más revelador son los rasgos triviales, los que habitualmente se desechan, se trata de desenmascarar. de seguir pistas, de detectar a través de lo accesorio, de encontrar alli donde el esfuerzo personal es más débil.

La conclusión a la que conducirá el razonamiento es un misterio para el que desconoce el método. $\mathrm{Si}$ los pasos intermedios no se hacen explícitos, el resultado se revela como algo asombroso para el no experto. El asombro que reflejan Watson o Aldo ante Sherlock Holmes o Guillermo de Baskerville es seguramente similar al que despertaba Beazley con sus acertadas atribuciones aparentemente incomprensibles y su prodigiosa memoria cuando observaba, por ejemplo, que un fragmento de Florencia pertenecía a un ánfora del pintor de Berlin en el Louvre ${ }^{20}$.

«La contribución real de Morelli al estudio del Arte fue que ideó un método mediante el cual las distancias entre la atribución y la obra de Arte podrian ser tan efectivamente salvadas que estariamos facultados para decir que es virtualmente cierto que una pintura concreta es la obra de un determinado artista. El argumento de Morelli es éste: todo artista verdadero está abocado a la repetición de ciertas formas o modos de hacer característicos» ${ }^{27}$. Las composiciones, la forma de colocar los personajes en la

\footnotetext{
${ }^{24}$ Sheperd, cit. (n. 23), págs. 19 y 22

${ }^{25}$ Sheperd, cit. (n. 23), págs. 22-23.

${ }^{26} \mathrm{D}$. von Bothmer, "Beazley the Teacher", en Beazley and Oxford, D. Kurtz (ed.), Oxford, 1985, p. 11.

${ }^{27}$ R. Wollheim, "Giovanni Morelli and the origins of Scientific Connoisseurships',, On Art and the Mind: Essays and Lectures, Londres, 1973, p. 177.
} 
escena, sus posturas y actitudes es algo que se realiza de forma consciente y más que algo propio de la individualidad del artista, pueden depender de corrientes artísticas concretas de un momento determinado. Pero los pequeños detalles, la manera de mover la mano al trazar una linea, de dibujar un ojo o una oreja es lo que analiza el método pues es lo que distingue la mano de un individuo de la de otro. «Beazley adoptó una versión del sistema de Morelli analizando la representación del detalle, especialmente anatómico, mediante el cual se puede detectar la "escritura" de un pintor, bien ya se repita la representación inconscientemente por lo trivial, o se adopte conscientemente y se mantenga. Lo trivial es a menudo lo más informativo.» $2 \mathrm{x}$

Lo primero es el análisis y conocimiento de la anatomía humana. En los vasos áticos y en el arte griego en general el tema es el tratamiento del cuerpo humano, vestido o desnudo. Hay que observar y conocer las distintas partes del cuerpo y conducirse con un método similar al de un forense o un botánico «buenos poderes de observación, y concentración, y razonamiento de la clase que supuestamente tienen el botánico o anatomista» ${ }^{20}$.

Como Morelli, Beazley analiza de forma continuada y detalladamente cada una de las partes del cuerpo, por ejemplo el ojo ${ }^{30}$, la oreja, o el tipo de pie $^{31}$. A veces el especialista utiliza para el análisis estilístico una terminologia que nos hace preguntarnos si estamos ante un trabajo sobre cerámica griega o hemos abierto un manual de anatomía ${ }^{32}$.

Beazley contrasta además el detallado análisis estilístico, casi de anatomista, de la figura humana, tomado del modelo de Morelli, con otros datos pro-

\footnotetext{
${ }^{2 \times}$ Boardman, cit., (nota 1), p. 8.

${ }^{24} \mathrm{Kurtz}$, cit. (n. 11) p. 246.

${ }^{30}$ P. e. en "Citharoedus" en 1922, p. 210 escribe: "The eye is usually composed of two lines, nearly but not quite straight. with a thin brown mark between them for the colour part of the eye, but the upper line of the upper lid is sometimes drawn as welln.

${ }^{31}$ En el mismo articulo continua p. 212 «the foot of the youth... is a good example of the type: notice the shape of the great toe and the other toes, and the black arcs making the ankles".

${ }^{32}$ P.e. en Kurtz: «Brown lines define details of the deltoid's musculature and a small arc the impression which this muscle's insertion creates about one third ot the length along the upper arm. Opposed brown arcs define contours of biceps and triceps, a small brown arc the tip of the elbow, three or more straightish lines the forearm's musculature. On the thumb-side one of these lines curves towards the bend of the arm. In the trunk, loops efect the transition from the chest, ovoids aproximate to the surface reliefs of rectus abdominis, doubled black arcs on the chest wall the interdigitations of serratus anterior with external oblique, and a black line on either side on the chest wall, above the doubled arcs, tje reliefs of latissimus dorsi" "Gorgos' cup: an essay in connoisseurship", JHS, CIII, 1983, p. 74.
}

pios de su material de estudio; como el análisis de las decoraciones vegetales, de las caras secundarias, las grecas o motivos que enmarcan las escenas, y observa no sólo el trabajo del pintor sino del alfarero: la forma del vaso. sus proporciones, etc. ${ }^{3}$. "Las formas de los vasos y sus decoraciones son guias exactas para la atribucion: los expertos, por lo tanto, combinan el análisis morelliano del dibujo con observaciones sobre el trabajo del alfarero y sobre el trabajo del decorador" ${ }^{3}$. La continua observación y el análisis de los elementos son los primeros componentes del método del "buen experto" pero falta algo: la comparación y la memoria visual. Una vez que se han aislado las caracteristicas concretas de un pintor es necesario confrontarlas con las de otros. La intuición se convierte en conclusión a partir de la observación de un gran número de detalles, lo que implica comparar un vaso con otro, un dibujo con muchos otros, con todos los que el investigador conozca. El "connoisseur» almacena todos los datos en su memoria, "Memoriza el sistema y pasea por el Louvre o el Museo Británico: no tendrás duda de en qué vasos está presente o en qué vasos está ausente» ${ }^{35}$.

Para atribuir es necesario observar todos los detalles. Son precisamente éstos, lo que se repite, lo secundario, el dibujo rápido e inconsciente, lo trivial, lo que ofrece información. Beazley observó también el dibujo de las caras secundarias, decoradas con las «mantle-figures», los jóvenes en himation que se repiten continuamente en las caras posteriores de muchos vasos de figuras rojas. Son escenas en las que se representan menos elementos y donde las lineas se reproducen con mayor regularidad. Incluso se siente obligado a defender el valor estilistico de estos jóvenes del reverso a los que dedica su atención, aunque Beazley seguía prefiriendo apoyarse en sus atribuciones en el análisis de la cara principal. Trata el tema por ejemplo en su estudio sobre el Pintor de Aquiles. Las figuras del reverso, observa, se repiten de la misma manera en distintos vasos pero prefiere dar la vuelta al vaso y comprobar que en la cara principal «las piernas, pies, manos, caras, vestidos están dibujados de la misma manera ${ }^{36}$.

Para desarrollar el método Beazley se apoya en la técnicas a su alcance: el dibujo y la fotografia. El dibujo era la técnica tradicional ya casi totalmente

\footnotetext{
"Beazley, cit. (n. 7), 1922, p. 85.

${ }^{34}$ B. B. Sparkes, Greek pottery, An Introduction, 1991, p. 113.

"Beazley, "Citharoedus", 1922, p. 83.

${ }^{36}$ Beazley. "The Master of the Achilles amphora in the Vatican», JHS, 34, 1914, p. 179.
} 
abandonada por la fotografia. Esta colma las ansias de objetividad cientifica, frente a la subjetiva interpretación de un dibujo. La fotografia se habia impuesto en la ejecución del corpus lasorum Antiquorum, frente a la tradicion monumental cuyo mejor ejemplo es la edición con dibujos de tamaño natural de A. Furtwängler y K. Reichold '. El CVA, impulsado por E. Pottier prefirió frente a la interpretación subjetiva y selectiva de la tradición monumental, objetivar, recoger, identificar, documentar a través de la técnica más objetiva de la fotografia ${ }^{\text {ix. }}$.

Beazley utiliza los dos métodos, pero para él era muy importante el valor de los dibujos en el estudio de la cerámica griega, tanto, que los terminaba aunque no tuviera intención de publicarlos y recomendaba para aprender a distinguir un estilo de otro, dibujar, dibujar la forma del vaso, la composición y sobre todo los detalles, dibujarlos más grandes que en el original; de esta manera, dice, el estudioso descubrirá muchos datos que se hubieran pasado por alto si no se hubiera dibujado ${ }^{34}$. En los dibujos debe primar la claridad y la exactitud intentando conseguir la máxima fidelidad y objetividad.

¿Cómo dibujar la decoración pintada de un vaso griego? D. von Bothmer distingue muy bien entre dos tipos de dibujo: el dibujo libre y los calcos ${ }^{+1}$. El mismo Bothmer nos explica que Beazley empezó utilizando el primero pero pronto se desanimó dado el tiempo que esto suponia y el frecuente mal resultado. Fue precisamente Karl Reichold quien, en 1908, cuando Beazley lo visitó en Münich, introdujo al joven en una técnica que resultaba totalmente nueva para él, el calco, que permite ajustarse a la superficie curva del vaso reproduciendo una escena completa que en una fotografia quedaría inevitablemente deformada y mutilada. De esta manera para Beazley el dibujo es una copia fiel, objetiva, del original. En el estudio de la cerámica griega es preferible sin duda a la fotografía. La fotografia no puede reproducir la escena completa en muchos vasos de curva pronunciada. A menudo en la reproducción fotográfica se pierden detalles, como el barniz diluído, 1932.

${ }^{17}$ Griechischen Vasenmalerei, Münich, I: 1904; II: 1919: III:

${ }^{7 \times}$ Sobre el origen y desarrollo del CVA, cf. R. Olmos, «El Corpus Vasorum Antiquorum, setenta años después: pasado, presente y futuro del gran proyecto internacional de la cerámica antigua», en $A E A, 62,1989$, p. 292-302.

${ }^{39} \mathrm{En}$ "The Training of Archaeologists", conferencia sobre el futuro de la Arqueologia, pronunciada en Londres en Agosto de 1943, publicada por D. Kurtz, Greek Vases. Lectures by J. D. Beazley, Oxford, 1989, p. 100-101.

"Dietrich von Bothmer, "The Executions of the Drawing". en The Berlin Painter. Drawings of Sir John Beazley Kurtz (ed), Oxford, 1983. los colores añadidos, perdidos cuando sólo se conserva la huella, etc. Es natural que Beazley, en sus notas personales prefiriese tomar algunos detalles o algunas figuras completas dibujadas. El estudioso se acerca asi al trabajo del pintor del Cerámico, casi con el mismo esfuerzo físico, reproduciendo cada linea, cada detalle. El trabajo, el proceso creativo del pintor griego, de cada pintor, se siente más próximo, y el estudioso puede captar detalles que de otro modo no advertiria. De alguna manera, a través del dibujo, el connoisseur "posee" el objeto representado. Beazley pone gran cuidado en la ejecución de sus dibujos, desde la elección del papel que en un principio adquiría en la misma tienda que Reichold, hasta la utilización de los lápices, más blando para las lineas en relieve o las fuertemente barnizadas, más duro y ligero para las lineas interiores, un lápiz oscuro para las inscripciones o rojo añadido, etc. ${ }^{+1}$.

Para llegar a tener un conocimiento profundo de la cerámica griega y llegar a convertirse en un «connoisseur» es necesaria la experiencia directa, el contacto físico con el material. Es necesario familiarizarse con el material y llegar a conocer los vasos y los estilos de los pintores tanto que el experto se sienta confortable con ellos, casi como si se tratara de viejos amigos ${ }^{42}$. Beazley educaba en este sentido a sus alumnos, como recuerda Bothmer en las clases del viernes en la casa del maestro. Al principio, durante el té, Beazley permanecía silencioso "obviously deep in thought", a las 5 llevaba a sus alumnos a su estudio adyacente y durante dos horas les enseñaba fotografias y fragmentos que les permitia tocar ${ }^{43}$. El profesor da unas reglas básicas, 10 mandamientos que expone en algo más de dos páginas ${ }^{44}$ donde recalca que hay que ocupar la mayor parte posible de tiempo observando los vasos directamente, no a través de fotografias o dibujos. El trabajo con fragmentos desarrolla y agudiza el sentido para diferenciar estilos.

El método que Beazley generalizó para la cerámica ática no está exento de riesgos. No todas las atribuciones propuestas, ni siquiera por Beazley son correctas. Siempre es posible que los detalles de un pintor que individualizamos y que nos conducen a una atribución sean copiados del maestro por los alumnos o se deban más que a dos artistas distintos

\footnotetext{
"Bothmer, "The executions of the Drawings", p. 6.

${ }^{42}$ Asi ve $\mathrm{M}$. B. Moore a M. Robertson en la recensión que hace de su libro: The art of vase-painting in Classical Athens, en American Journal of Archaeology, 98. 1994, p. 170.

${ }^{43}$ Bothmer, "Beazley, the Teacher", en Beazley and Oxford. D. Kurtz (ed), Oxford, 1985, p. 6.

${ }^{44}$ Beazley, cit. en (nota 38), p. 98.
} 

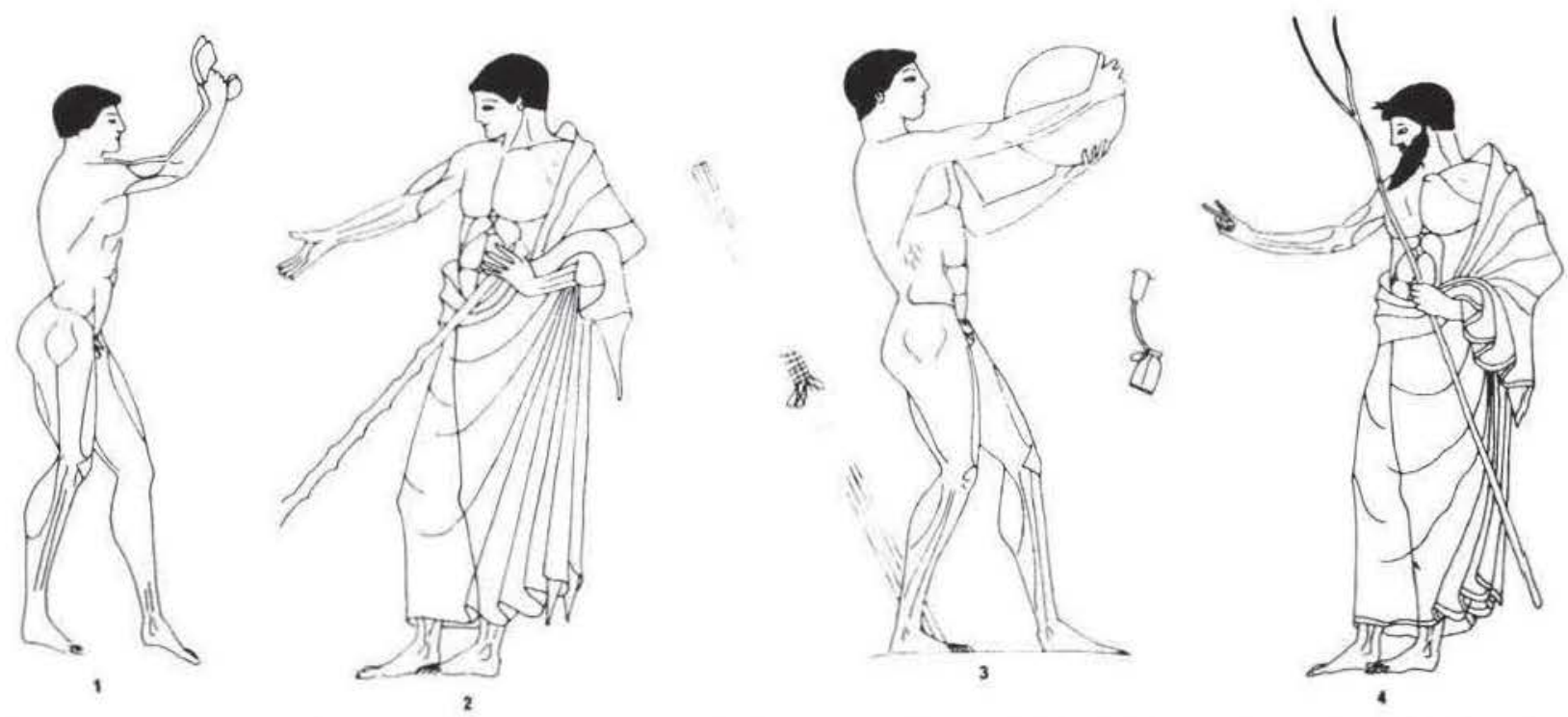

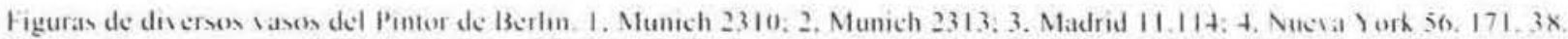

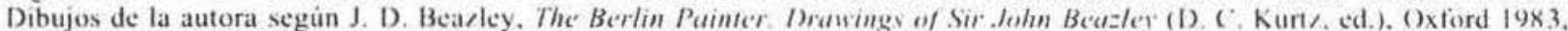
lams. I. II y VIII.

a cambios introducidos por un viejo pintor, o se pueden confundir artistas estrechamente ligados. Todo esto lo reconoce el propio Beazley ${ }^{\text {t5 }}$. Aunque, sin duda, la mayor parte de lo atribuido por Beazley fueron aciertos. El método morelliano de atribución se presta mejor al análisis del dibujo de lineas de la cerámica griega que al de los trazos de pincel de la pintura italiana. Los vasos griegos bien conservados son numerosos en todos los museos importantes y nos han llegado prácticamente intactos, sin repintes, que por otra parte se pueden detectar fácilmente lo que es otra ventaja frente a la pintura al fresco o tempera ${ }^{\text {th }}$. Una de las mayores aportaciones de Beazley fue su extensión del método, que aplicó no sólo a los vasos de gran calidad, sino a toda la cerámica griega, hasta la más humilde. Beazley, heredero del pensamiento de fines del xix, creó un método de trabajo seguido y continuado por muchos otros, una forma de trabajar «que ha condicionado fuertemente nuestra manera de aprehender la cerámica ática» ${ }^{47} \mathrm{y}$ el arte griego en general.

"V. prefacio de Atric Red-figured Vases in American Museu$m s$, Cambridge, 1918, reed. por L'Erma di Bretschneider, Roma, 1975.

th Cf. B. Ashmole, "Sir John Beazley (1885-1970)", en Beazley and Oxford, Oxford, 1985, p. 65.

${ }^{47} \mathrm{~F}$. Frontisi-Ducroux y F. Lissarrague "Vingt ans de vases grecs. Tendances actuelles des études en iconographie grecque», Metis, V. 1990, p. 205.

\section{UNA CUESTIÓN DE EXPERTOS}

«El éxito incondicional de Beazley, autoridad incontestable, y su desgana para explicar por escrito cómo miró los vasos griegos, encubrió las sutilezas $-\mathrm{y}$ peligros - de la atribución para muchos arqueólogos clásicos que ahora esperan una atribución de algún "especialista en vasos»" ${ }^{4 \times}$.

El razonamiento detectivesco de Beazley y de otros expertos, cuyos pasos no son explicados, y cuyos resultados se ofrecen como algo casi incuestionable, se presentan como incomprensibles al profano. como algo sorprendente, aunque para el investigador que conoce el método la conclusión sea «elemental». Esto produjo un efecto sin duda no deseado por Beazley. Las atribuciones de los expertos y mucho más las del maestro de Oxford han sido repetidas mecánicamente, $\sin$ critica, $\mathrm{y}$ tomadas como dogma. Aunque uno de los méritos de Beazley es hacer posible que el método sea utilizado por otros también, lo cierto es que debido en gran parte a que no explicó su método por escrito, la atribución se ha convertido en algo de y por especialistas. Esta cuestión de expertos, no argumentada, conserva un aire misterioso donde se erige casi como único argumento el criterio de autoridad. Esta es una de las

\footnotetext{
${ }^{4 k}$ Kurtz. "Gorgos" cup: an essay in connoisseurship", cit
} (n. 32 ), p. 69. 
criticas mas importantes. la utilizacion del metodo está unida al autoritarismo. a una frectente renuncia a la argumentacion racional y apela a la reputacion personal ".".

La exclusividad del método se acentua con la muchas veces incomprensible lectura de la terminologia utilizada. Se emplean. cuando se atribuye un vaso, términos ambigüos como "cercano a», "próximo a", "a la manera de», "del grupo de", "del circulo de", "seguidores de", etc. cuyas fronteras son difusas y sus explicaciones en ocasiones cuando las hay terriblemente subjetivas y dificiles. Esta terminologia poco definida puede encerrar en algún caso, escondiéndolas, las dudas de los propios expertos. Asi un vaso - o un fragmento- puede estar hecho «a la manera de» o ser "próximo a» un pintor al que nos ha faltado el valor, la seguridad o la decisión de atribuirlo sin reservas.

Los argumentos que apoyan las atribuciones y el lenguaje es muchas veces impreciso, por ejemplo G. Richter afirma que un vaso está hecho «a la manera de" un pintor porque "las graciosas figuras y atractivas composiciones se derivan directamente de las creaciones del Pintor de Meidias, pero no tienen el toque delicado del maestro"; o un vaso se atribuye a un grupo porque «el delicado, amplio estilo del dibujo, sitúa la pintura en el grupo del pintor de Jena» ${ }^{50}$, por citar sólo algunos ejemplos de una forma frecuente de escribir. Las dificultades en comprender la terminología no se reducen sólo a los no iniciados sino también a algunos estudiosos. ¿Cómo delimitar y definir una "atractiva composición» o "un toque delicado»? Boardman nos dice que «las relaciones entre pintor $\mathrm{y}$ alfarero, $\mathrm{y}$ los criterios que definen la relación maestro-alumno o de taller son a veces claras, a veces no. Beazley encontró necesario indicar una distinción entre un vaso de un pintor y un vaso a la manera de ese pintor; y que «manera», «imitación», «seguidor», «escuela», "círculo», «grupo», «influencia», «relación» no son, en mi vocabulario, sinónimos. No todos los estudiosos son capaces de seguir todas estas distinciones" ${ }^{51}$. Pero ni Beazley ni Boardman dicen claramente cuáles son esas diferencias.

Pero en general en el uso de un vocabulario más

\footnotetext{
${ }^{49} \mathrm{~V}$. Gaskell, Historia de las imágenes, en P. Burke (ed.). Formas de hacer historia, Alianza Universidad, Madrid, 1993 (Trad. de New Perspectives on Historical Writing. Polity Press. 1991), págs. 216 y ss.

${ }^{50} \mathrm{G}$. Richter, Attic Red-Figured Vases. A Survey, New Haven, Yale Un. Press, 1946, p. 149.

"1. Boardman, Athenian Red Figure Vases: The Archaic Period, 1975, págs. 8 y 9.
}

preciso y comprensible para el estilo de un pintor tambien se ha avanzado. Se intentan enumerar de una forma sencilla y clara, abandonando metáforas poéticas, las caracteristicas de un pintor. Basta leer la bibliografia de los últimos años, las sintesis de Boardman o de Robertson que intentan ser inteligibles y completas 5 .

\section{DESPUÉS DE BF:AZLEY}

Hace ya casi 25 años, el 9 de mayo de 1970, que murio John Beazley. Su obra transformó profundamente nuestro modo de ver la cerámica griega. Desde su muerte muchos han consolidado y continuado su obra publicando numerosas monografias de pintores ya identificados por el maestro ${ }^{53}$. Con estos trabajos las listas de pintores de Beazley se hacen visibles con ilustraciones de los vasos, pero salvo excepciones, no se trata más que de una continuación en la misma línea ${ }^{54}$ con una extensa bibliografía especializada $^{55}$. Desde 1980 los especialistas se reúnen en unos coloquios sobre cerámica griega que se celebran regularmente, y que comenzaron con la intención de organizar el trabajo después de Beazley ${ }^{56}$. Las investigaciones de la Universidad de Oxford derivan aún en gran medida de la línea iniciada por Beazley. Tras su muerte en 1970 se trasladó el archivo del profesor con las fotografias, las notas y los dibujos al Ashmolean Museum ${ }^{57}$. Donna Kurtz

"J. Boardman: Athenian Blak Figure Vases, Londres, 1974: Athenian Red Figure Vases: The Archaic Period, Londres. 1975; Athenian Red Figure Vases: the Classical period. Londres, 1989: M. Robertson. The art of vase-painting in classical Athens, Cambridge, 1992.

${ }^{51}$ G. Bakir, Sophilos. Ein Beitrag zu seinem Stil, 1981; en la serie Kerameus: H. Momsen, Der Affecter, 1975; A. Lezzi-Hafter, Der Schuvalov Maler, 1976; E. Böhr. Der Schaukelmaler. 1982; A. Lezzi-Hafter, Der Eretria Maler. 1988; J. Burow. Der Antimenes Maler, 1990; J. Oakley. The Phiale Painter, 1990; L. Burn, The Meidias Painter, Oxford, 1982. Casi inexistentes siguen siendo las monografias dedicadas a los pintores más tardíos, sin embargo podemos citar el trabajo de S. Drougou: «Erythrómorphos cratiras tou $40 u$ eóna apó ti Véria. O sográfos tís Toya». Archeologiki Ephimeris, págs. 85-98.

${ }^{54}$ V. F. Frontisi- Ducroux y F. Lisarrague, cit. (n. 47), págs. 209 y ss.

" $C f$. los resúmenes de $\mathrm{H}$. Metzger en Bulletin Archéologique de la Revue des Études Grecques, 1972. págs. 83-138: 1973. págs. $344-411$; 1976 , págs. $361-414 ; 1978$, págs. $511-554 ; 1980$ págs. $136-185 ; 1982$, págs. $85-139 ; 1984$, págs. $135-188 ; 1986$, págs. $63-116 ; 1989$, pảgs. $58-123 ; 1990$, págs. $617-668$.

so Vasenforschung nach Beazley (Tübingen, 1978), Mayence, 1979. El siguiente no se celebró hasta 1984: Ancient Greek and Related Pottery. H. A. G. Brijder (ed.), Amsterdam. El último fue en 1987 en Copenhague: 3. Symposium on Ancient Greek and Related Pottery. J. Christiansen y T. Melander (eds.), Copenhague, 1988.

"Boardman, en Beazley and Oxford. 
no solo es su archivera sino que gracias a su trabajo ha convertido este lugar en el centro mundialmente más importante para el estudio de la cerámica ática pintada. En 1979 se puso en marcha un plan para informatizar el archivo y publicar addenda a las listas de Beazley".

A pesar del éxito. o precisamente por ello, el método sigue recibiendo criticas. Se ataca tanto su validez como la aproximación al estudio de los vasos griegos adoptada por Beazley. Algunos critican la arbitrariedad del método mismo. Desde 1937 que recibió ya esta critica de E. Pottier " hasta nuestros dias, no ha dejado de ser atacado por esta razón. Boardman lo defiende y afirma que el método "puede no satisfacer a la nueva Arqueologia. pero es necesario que no nos avergoncemos de poder usar nuestros ojos como nuestros instrumentos. La coherencia de un grupo de vasos atribuido a una mano por Beazley puede ser demostrada por el análisis computerizado de detalle" ${ }^{60 t}$. Robertson también defiende el método de Beazley aunque su entusiasmo no le impide reconocer que algunas de las atribuciones de Beazley, sobre todo las que se refieren a artistas menores, pueden ser discutidas, aunque otras, como los vasos adscritos al Pintor de Berlin o al Pintor de Pan son tan inconfundibles a sus ojos como los grabados de Durero o Utamaro ${ }^{\circ 1}$.

Ciertamente el porcentaje de aciertos de Beazley fue espectacular. Pero naturalmente el acierto depende de la capacidad del experto. No todos poseen los ojos de Beazley, su increible capacidad y memoria. Es necesario que asumamos la arbitrariedad del método, la posibilidad de error y es conveniente que los estudiosos usen una terminología clara y precisa y expliquen el por qué han llegado a una atribución dada, separando los criterios estilisticos de los que no lo son. El camino puede ser desandado por otro más fácilmente. A pesar del riesgo, sigue siendo útil explotar la posibilidad que se abrió con Beazley de relacionar un vaso con un taller o pintor concreto y su método es el único medio de que disponemos, no existe alternativa, es una acti-

sx Continuando y completando la obra de Beazley se han publicado dos Beazley Addenda: Additional References to $A B V$. ARV2 and Paralipomena, compilado por Lucilla Burn y Ruth Glynn. Oxford, 1982 y Thomas H. Carpenter, Oxford, 1989. Sobre el archivo Beazley en el Ashmolean v. p.e. D. Kurtz, aLa base de datos del archivo Beazley' AEA, 64, 1991, pág. 366.

${ }^{40} \mathrm{~V}$. F. Frontisi-Ducroux y $\mathrm{F}$. Lissarrague. "Vingt ans de vases grecs...m, p. 205.

"Boardman, Athenian Red Figure Vases: The Archaic Period, 1975 , p. 8.

"Robertson, The art of vase-painting in Classical Athens. Cambridge, 1992, p. 4. lidad aún necesaria si bien no en cuanto fin en si misma sino como medio para obtener una gran variedad de resultados.

Otra linca de ataque no se refiere al método en si sino a su utilización por los especialistas, una consecuencia sin duda no deseada por Beazley, Lo sintetiza bien Hoffimann: "El influjo de Bearley y de la escuela de Oxford acentuará lo que desde ángulos de observación más externos se ha denominado la mania de la atribucion a talleres y pintores, que se ha convertido tal vez exageradamente en un fin primordial del experto en vasos griegos" "2. Beazley y sobre todo sus seguidores nos han hecho creer que lo más importante de cualquier vaso griego es quién lo ha pintado. La mania de la atribución ha perjudicado, a mi juicio, el avance del estudio. Muchos expertos han tomado como dogma las listas de Beazley y se han preocupado bien de ampliarlas con nuevos vasos o bien de fraccionarlas. multiplicando el número de pintores ${ }^{\text {"t }}$ como todo avance. No se concibe un catálogo de vasos griegos sin su correspondiente atribución, aunque es frecuente que no se preste atención a otros aspectos del vaso, como sus imágenes, sus procedencias, etc. Es natural, pues, que en los ultimos años muchos estudiosos - particularmente los procedentes de campos estructuralistas o antropológico-sociológicos-, ataquen tanto el método en si como la aproximación desde esta óptica al estudio de la cerámica griega ${ }^{64}$.

Robertson, que defiende con entusiasmo el método de Beazley, tiene la impresión de que todas estas manifestaciones de descontento tienen algo que ver con la aplicación de un método tomado de la Historia del Arte a producciones artesanales no realmente artisticas ${ }^{\circ 5}$. Cree "que la premisa de la que parte Beazley ignora una profunda diferencia entre las dos manifestaciones artisticas, y este error para reconocer esta diferencia ha tenido malas consecuencias para el estudio" ${ }^{66}$ y que "fue el movimiento de artes y oficios de finales del xix, un movimiento fundado en parte en el puritanismo, en parte en una reacción contra los métodos de producción mecanizados, lo que dió a la cerámica el estatus de arte.

"2:Herbert Hoffmann. «In the Wake of Beazleyn, Hephaistos. 1. 1979, págs, $60-71$.

${ }^{6} \mathrm{~F}$. Frontisi-Ducroux y F. Lissarrague, cit. (n. 47), p. 209 con bibliografia.

th Algunas de estas criticas y la defensa al método en Robertson, cit. (n. 61 ), págs. 4 y ss.

${ }^{45}$ Robertson, cit. (n. 61), p. 5.

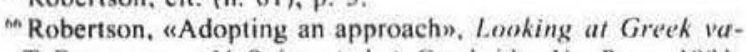
ses, T. Rasmussen y N. Spivey (eds.), Cambridge Un. Press, 1991. p. 2 . 
Beazley, llevado por esta atmósfera llevó este coneepto hasta los griegos, que habrian reido ante la idea de que su cerámica fuera tan estimada; y haciendo esto Beazley distorsionó nuestra visión del arte griego»". Esta discusión estéril sobre si se deben considerar o no producciones artisticas los vasos griegos creo que está en nuestros dias superada. Lo que si se critica a Beazley es haber tomado el método de los estudios de pintura italiana y haberlo aplicado, quizá demasiado mecánicamente al principio, a producciones muy distintas. Esto le lleva a utilizar una aproximación al estudio y una terminología poco apropiada como hemos señalado antes. Parece que Beazley veia el funcionamiento de los talleres del Cerámico como los estudios de los pintores italianos donde aprendices y asistentes producian obras de los diseños del maestro. Como señala Robertson, por lo que sabemos del funcionamiento de los talleres de Atenas, esto no era asi. Cada taller debía tener uno o dos decoradores principales y otros que aprendian el oficio, en algunos casos parece que se producia una relación maestro-alumno - p.e. en el caso del Pintor de Berlín, Pintor de Aquiles, Pintor de la Phiale ${ }^{\text {ck. }}$.

Debido al enorme peso de Beazley que centró toda su atención en el estudio de la cerámica figurada, la balanza se ha descompensado y la cerámica ática se ha dividido artificialmente en dos grandes bloques: la figurada y la barnizada, y su estudio es abordado muchas veces por especialistas distintos. Los trabajos siguen caminos paralelos pero sin unirse, a pesar de que eran producciones de los mismos talleres. Es necesario abordar el estudio conjunto de la cerámica ática $\sin$ despreciar las producciones barnizadas, hay que buscar la relación entre ambas, relación que podemos encontrar en el análisis de los

\footnotetext{
". Robertson, "Beazley and Attic Vase Painting", Beazley and Oxford, Oxford, 1985, p. 20.

".x Robertson, "Beazley and Attic Vase Painting", p. 27. y id. The art of... p. 5 .
}

datos arqueológicos ${ }^{\text {tho. }}$. Hay que superar la pura atribución sin negar su utilidad y no despreciar otras aproximaciones al estudio que pueden ser simultáneas.

Es necesario también ser conscientes de los puntos débiles del método. Las atribuciones son inevitablemente subjetivas. El experto deberia asumir la arbitrariedad del método y hacer aparecer sus atribuciones como inciertas, evitando asi el peligro de lo que se da como auténtico. La inseguridad nos lleva a argumentar, a explicar, a dibujar, mientras que la atribución "segura», y misteriosa, podría en algún caso conducir al experto $\mathrm{y}$ a sus lectores por un camino falso. Difícilmente podrán salir de él si desde la entrada no se ha empezado a desenrrollar el ovillo.

A pesar de las comprensibles criticas, el método es válido y uno de los méritos de Beazley es habernos salvado de una visión estrecha y reducida de la cerámica griega tal como tenemos, por ejemplo, del arte prehelénico. Podemos seguir la manera en la que el arte griego se ha desarrollado casi durante 300 años si no con nombres propios, si con personalidades concretas. Gracias a eso conocemos mejor la evolución de la cerámica que la de la escultura u otros objetos menores. El estudio estilistico y la atribución es un medio por el que podemos llegar a conocer con gran precisión cuestiones no sólo de evolución artística sino históricas, iconográficas, etc. Debemos tener siempre presente los resultados a los que nos encaminamos y que queremos obtener. «Debemos elegir la descripción que estipulemos que nos da historia y leer a partir de ella" ${ }^{70}$. El método, como todos los métodos, tiene en sí mismo una validez. relativa. Lo importante son los resultados que obtengamos a partir de él y la calidad de estos resultados.

\footnotetext{
${ }^{\circ} \mathrm{V} V$. p.e. mi trabajo "La cerámica ática de Andalucia en el siglo Iv. El «taller del Pintor del Tirso Negro". Huelva Arqueológica, 1994.

${ }^{70} \mathrm{~W}$. Davis, "Style and history in art history", en The uses of style in archueology. New directions in Archaeology, M. Conkey y C. Hastorf (eds.), Cambridge, 1990, p. 30.
} 